\title{
Effect of orthodontic treatment on alveolar bone thickness in adults: a systematic review
}

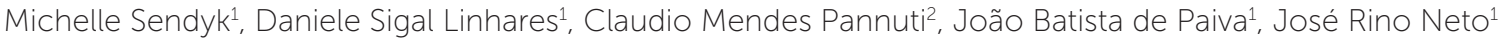

DOI: https://doi.org/10.1590/2177-6709.24.4.034-045.oar

Objectives: This review aimed at evaluating changes in alveolar bone thickness after completion of orthodontic treatment. Methods: Only prospective clinical studies that reported bone thickness in adult patients undergoing non-surgical orthodontic treatment were considered eligible. MEDLINE, EMBASE and LILACS databases were searched for articles published up to July 2018. Results: A total of 12 studies met the selected criteria. Most of the studies showed that orthodontic treatment produces a reduction in bone thickness of incisors, mainly at the palatal side. Conclusion: On patients undergoing different orthodontic treatment techniques, there was a significant bone thickness reduction, mainly on the palatal side. Clinical relevance: These findings are relevant and have to be considered in diagnosis and planning of tooth movement, in order to prevent the occurrence of dehiscence and fenestration in alveolar bone.

Keywords: Orthodontics. Alveolar bone loss. Periodontics. Tooth movement.

Objetivo: o objetivo da presente revisão foi avaliar as mudanças na espessura óssea alveolar após o término do tratamento ortodôntico. Métodos: as bases de dados MEDLINE, EMBASE e LILACS foram consultadas para artigos publicados até julho de 2018, sendo considerados elegíveis somente estudos prospectivos clínicos que relataram a espessura óssea em adultos submetidos ao tratamento ortodôntico não cirúrgico. Resultados: no total, 12 estudos atenderam aos critérios de inclusão, e a maioria dos estudos mostrou que o tratamento ortodôntico promove redução na espessura óssea dos incisivos, principalmente na face palatina. Conclusão: em pacientes submetidos a diferentes técnicas ortodônticas, foi observada uma redução significativa na espessura óssea, especialmente na face palatina. Relevância clínica: esses achados são relevantes e devem ser considerados no diagnóstico e plano de tratamento do movimento dentário, com o objetivo de prevenir a ocorrência de deiscências e fenestrações ósseas.

Palavras-chave: Ortodontia. Perda óssea alveolar. Periodontia. Movimento dentário.

${ }^{1}$ Universidade de São Paulo, Departamento de Ortodontia e Odontopediatria, Divisão de Ortodontia (São Paulo/SP, Brazil).

${ }^{2}$ Universidade de São Paulo, Departamento de Estomatologia, Divisão de Periodontia (São Paulo/SP, Brazil).
How to cite: Sendyk M, Linhares DS, Pannuti CM, Paiva JB, Rino Neto J. Effect of orthodontic treatment on alveolar bone thickness in adults: a systematic review. Dental Press J Orthod. 2019 July-Aug;24(4):34-45. DOI: https://doi.org/10.1590/2177-6709.24.4.034-045.oar

Submitted: January 17, 2018 - Revised and accepted: March 07, 2019

" The authors report no commercial, proprietary or financial interest in the products or companies described in this article.

Contact address: Michelle Sendyk

Universidade de São Paulo, Departamento de Ortodontia

Av. Professor Lineu Prestes, 2227, Cidade Universitária

E-mail: msendyk@usp.br - São Paulo/SP - CEP: 05.508-000 - Brazil 


\section{INTRODUCTION}

The longevity of a tooth depends on its periodontal health. Evidences show that orthodontic treatment can result in loss of periodontal support in the presence of plaque and inflammation. ${ }^{1-3}$ Orthodontic treatments that result in pronounced tooth inclinations are considered to be risk factors for dehiscence and fenestration. One possible factor related to these occurrences is the reduced thickness of the alveolar bone around the roots. ${ }^{4}$ Thus, it is important to treat with caution orthodontic patients who already have thin soft-tissue margins before treatment, since the buccal tooth movement may render the gingival tissue more vulnerable and less resistant to plaque and tooth brush trauma. ${ }^{4-13}$

The first attempt to delineate the effect of tooth movement on bone thicknesses concentrated on animal studies. ${ }^{14,15}$ Subsequently, human studies were conducted using lateral and frontal cephalometric radiographs. ${ }^{16,17}$ However, the radiographic methods are affected by the superimposition of anatomical structures, difficulties in identification of individual teeth and magnification errors. ${ }^{18}$

Currently it is possible to measure alveolar bone thickness around the roots using the images obtained by cone beam computed tomography (CBCT). ${ }^{19-22}$ The accuracy and reproducibility of CBCT are well documented in the literature. ${ }^{23,24}$ However, to our knowledge, no systematic review has evaluated the effects of orthodontic treatment on bone thickness using CBCT.

Thus, the aim of the present systematic review (SR) is to evaluate the effects of orthodontic treatment on alveolar bone thickness, comparing different types of treatment techniques in adult patients.

\section{MATERIAL AND METHODS}

\section{Search strategy}

The study protocol of this SR was registered at the National Institute for Health Research PROSPERO (International Prospective Register of Systematic Reviews, http://www.crd.york.ac.uk/prospero). The review text was structured in accordance with guidelines from PRISMA (Preferred Reporting Items for Systematic Reviews and Meta-Analyses) and the Cochrane Handbook of Systematic Reviews of Interventions.
Search strategies were developed for MEDLINE via PubMed, EMBASE and LILACS databases until July 2018. MesH terms and keywords were combined with Boolean operators and used to search the databases:

\#1: (tomography OR cone beam computed tomography OR tridimensional OR CBCT OR cone-beam);

\#2: (bone thickness OR alveolar thickness OR alveolar bone OR fenestration OR dehiscence OR width);

\#3: (orthodontic OR malocclusion);

(\#1 AND \#2 AND \#3).

After the initial electronic search, the authors manually searched articles in the bibliographies of the included studies.

\section{Inclusion and exclusion criteria}

Only randomized clinical trials, controlled clinical trials, case series and observational prospective studies with one or more orthodontic treatment arms and tridimensional evaluation of alveolar bone thickness before and after the orthodontic treatment in adult patients were considered eligible for inclusion in this review. Observational studies that included children, patients who had received orthopedic rapid maxillary expansion or accelerated orthodontic treatment such as perforation or corticotomies; studies in which bone thickness was not evaluated using CBCT and studies performed in patients with syndromes and cleft patients were excluded from the review. Further, animal studies, letter to the editors, reviews and in vitro studies were not included.

Different techniques of corrective orthodontic treatment were considered for this review, among them Straight-wire and Edgewise techniques, and extraction and non-extraction treatments.

The primary outcome was alveolar bone thickness change. The alveolar bone thickness was measured on maxillary or mandibular central and lateral incisors, upper canines, upper and lower premolars and evaluated at three different distances $(3,6$ and $9 \mathrm{~mm}$ ) from the cementoenamel junction (CEJ) (cervical, middle or apical).

\section{Data extraction}

Data were extracted independently by two reviewers, and the disagreements were solved by discussion with a third reviewer. Studies appearing to meet the inclusion criteria or those with insufficient information in the title and abstract to allow a clear decision 
were selected for assessment of the full text, which was carried independently by the same two reviewers to determine study eligibility. Studies that met inclusion criteria underwent a validity assessment and data extraction. The reason for rejecting studies were recorded for each study.

Data were extracted and recorded using extraction forms. ${ }^{25}$ The following variables were assessed: 1) type of study, 2) characteristics of the participants, including definition of malocclusion, 3) follow-up duration, 4) characteristics of the intervention, 5) sample size, 6) outcome measures, 7) method of randomization, 8) blindness of examiners, and 9) source of funding and conflicts of interest.

\section{Risk of bias}

Risk of bias of the included studies was evaluated according to the Cochrane Collaboration's Tool for Assessing Risk of Bias. Briefly, the randomization and allocation methods (selection bias); completeness of the follow-up period/incomplete outcome data (attrition bias); blinding of patients (performance bias) and examiners (detection bias); selective reporting (reporting bias); and other forms of bias were classified as adequate (+), inadequate (-), or unclear (?). Based on these domains, overall risk of bias was categorized as follows: (1) low risk of bias if all criteria were met (adequate methods of randomization and allocation concealment, a "yes" answer to questions about completeness of follow-up and blinding, and a "no" answer to selective reporting and other sources of bias); (2) unclear risk of bias if one or more criteria were partially met; or (3) high risk of bias if one or more criteria were not met.

The methodological quality of the observational studies was assessed using the Newcastle-Ottawa scale (NOS).

\section{RESULTS}

\section{Articles}

Initially, 491 references were electronically selected. No additional article was manually identified. After title and abstract evaluation, 436 papers were excluded. The full texts of the remaining 55 publications were considered for detailed reading. Of these publications, 12 were considered eligible for inclusion (Fig 1).

\section{Included studies}

The characteristics of the included studies are shown in Table 1. From the 12 selected studies, two randomized controlled clinical trials, ${ }^{26,27}$ three controlled clinical trials, ${ }^{28-30}$ and seven case series ${ }^{31-37}$ were found. The studies were conducted in South Korea, ${ }^{31}$ Brazil, ${ }^{26,29,37}$ Denmark, ${ }^{27}$ Italy, ${ }^{28}$ India, ${ }^{32}$ Turkey, ${ }^{33}$ China, ${ }^{30}$ Thailand, ${ }^{34,36}$ and United States. ${ }^{35}$ Research foundations or universities supported three studies. ${ }^{30,34,36}$ A software company supported one study. ${ }^{27}$ None of the studies reported the follow-up period. A total of 291 orthodontic patients were included in the studies. In the selected articles various types of orthodontic treatment were evaluated and compared. Straight wire appliances with self-ligated and conventional brackets were compared. ${ }^{26}$ Among the different types of self-ligated brackets, passive and active appliances were compared. ${ }^{27}$ Some articles reported orthodontic treatment with Edgewise appliances. ${ }^{28,29,32,37}$ Among the different types of orthodontic treatment, the treatment featuring extraction of the upper first premolars were compared with treatment without extractions. ${ }^{28,29}$ Seven studies reported the periodontal status of the patients prior to orthodontic treatment and excluded patients with periodontal disease. ${ }^{27,31-36}$

\section{Quality assessment}

Among the 5 clinical trials, only one reported an adequate method of randomization. ${ }^{27}$ None of the trials reported an adequate method of allocation concealment. Only one article described blinding of examiners on treatment procedures. ${ }^{27}$ No study mentioned blinding of participants. The number of patients at baseline and final examination was described in three articles. ${ }^{26-28}$ Therefore, based on the criteria established by the present review, three studies ${ }^{28-30}$ were considered to present a high risk of bias and two studies $^{26,27}$ were considered to have unclear risk of bias (Fig 2). Furthermore, only one trial ${ }^{26}$ reported sample size calculation.

In the 7 case series, the Newcastle-Ottawa scale (NOS) was used to verify methodological quality (Tab 2). NOS scale was adapted for the purpose of this review, and each included study received a maximum of 14 points. Studies with 9-14 points were considered as presenting high methodological quality; 6-8 points studies, as medium quality; and those 


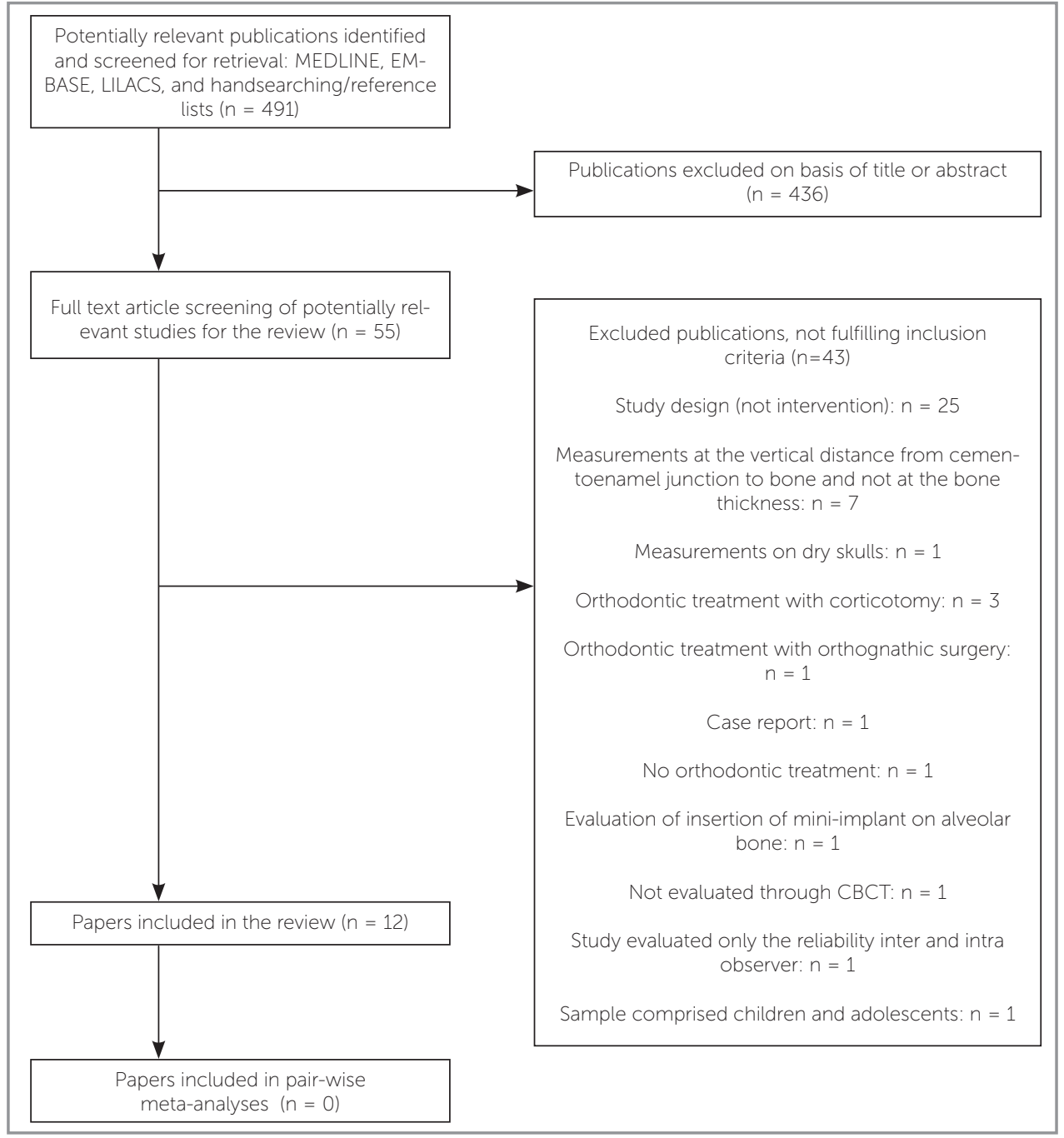

Figure 1 - PRISMA diagram of article retrieval. 
Table 1 - Characteristics of the studies.

\begin{tabular}{|c|c|c|c|}
\hline Study & Country & Study design & Follow-up \\
\hline Ahn et al. ${ }^{31}, 2013$ & South Korea & Case series & Not reported \\
\hline Almeida et al. ${ }^{26}, 2015$ & Brazil & $\mathrm{RCT}$ & Not reported \\
\hline Cattaneo et al. ${ }^{27}, 2011$ & Denmark & Parallel RCT & Not reported \\
\hline Lombardo et al. ${ }^{28}, 2013$ & Italy & Controlled clinical trial & Not reported \\
\hline Nayak-Krishna et al.32, 2013 & India & Case series & Not reported \\
\hline Oliveira et al. ${ }^{37}, 2016$ & Brazil & Case series & Not reported \\
\hline Picanço et al. ${ }^{29}, 2013$ & Brazil & Controlled clinical trial & Not reported \\
\hline Sarikaya et al. ${ }^{33}, 2002$ & Turkey & Case series & Not reported \\
\hline Sun et al. ${ }^{30}, 2015$ & China & Controlled clinical trial & Not reported \\
\hline Thongudomporn et al. ${ }^{34}, 2015$ & Thailand & Case series & Not reported \\
\hline Uribe et al. ${ }^{35}, 2013$ & USA & Case series & Not reported \\
\hline Yodthong et al. ${ }^{36}, 2013$ & Thailand & Case series & Not reported \\
\hline
\end{tabular}

with $<6$ points, as presenting low methodological quality. Of the seven included studies, two received a 7-point score, ${ }^{31,32}$ one a 3-point score ${ }^{33}$ and four a 6-point score $^{34-37}$ (Table 2). Thus, 6 studies were considered as medium methodological quality and 1 as low methodological quality. Two studies reported sample size calculation, ${ }^{34,37}$ and no study gave information about training of assessors, comparability of groups on the basis of the design, assessment of clinical conditions and adequacy of follow-up patients.
In all of the included studies, ascertainment of the bone before orthodontic treatment and validity of statistical analysis were considered adequately addressed.

\section{Effects of interventions Different types of treatment}

Twelve studies assessed changes in bone thickness as a result of the orthodontic movement ( $\mathrm{Ta}-$ ble 3). Five trials showed a significant reduction in bone thickness associated with retraction of anterior 
Sample size (baseline)

$n=37$ female

Age range: $26.6 \pm 8.5$ years

$\mathrm{n}=25$ (sex distribution not mentioned)

Age mean (years): $18.58 \pm 5.43$ (test):

$21.61 \pm 6.69$ (control)
CBCT Specifications

Implagraphy, 12×9-cm field of view, 90-kVp, 4.0-mA tube current,

$0.2-\mathrm{mm}$ voxel size and 24-second scan time

i-CAT Imaging Sciences International, 22x16-cm field of view, $120 \mathrm{kVp}$,

$36 \mathrm{~mA}, 0.4-\mathrm{mm}$ voxel size and 40-second scan time

CMF Software (M.E. Muller Institute for Surgical

Technology and Biomechanics, University of Bern, Switzerland, developed under the funding of the CO-ME Network)

NewTom 3G, 12 in field of view, 0.36-mm voxel size

Source of funding

No

Age mean (years): $16.0 \pm 5.7$ (test); $15.0 \pm 3.3$ (control)

NewTom 3G, 12 in field of view, 110- kV, 2.00 mA, 5.4 second exposure time

No Age mean (years): 11.9 (test); 10.11 (control)

GE medical systems, 120 kV, 160 mva

No

Age range: $15 \pm 3$ years

i-CAT Imaging Sciences International, 13x17-cm field of view, $120 \mathrm{kVp}$, $5 \mathrm{~mA}, 0.4-\mathrm{mm}$ voxel size and 20 -second scan time

No

Age range: 18 to 26 years old

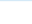

$\mathrm{n}=12$ (10 male and 2 female)

Age mean: $15.83 \pm 4.87$ years (test);

Not reported

No

$18.26 \pm 6.42$ years (control)

$n=19$ (sex distribution not mentioned) Age mean: $14.1 \pm 2.3$ years

Tomoscan SR7000, $120 \mathrm{kV}, 175 \mathrm{~mA}$ and 1.5-mm slice thickness

No

$n=42$ (sex distribution not mentioned) Age mean: not mentioned
Galileo, 150-mmx150-mm field of view, 85 kV, 21 mA, 20 second exposure time

This work was supported by the School Funds of Jinling Hospital, School of Medicine,

Nanjing University (No. 2013079). Open

Science Foundation for National Key Laboratory of Military Stomatology (No. 2014KC02), and China Postdoctoral Science

Foundation (No. 2015M572814)

Grant support from Graduate School and the Faculty of Dentistry, Prince of Songkla University

Age mean: $9.9 \pm 1.0$ years

Veraviewepocs J Morita MPG, 80 kV, 5 mA, 7.5 second exposure time, $0.125 \mathrm{~mm}$ voxel resolution, $80 \times 40 \mathrm{~mm}$ field of view

i-CAT Classic scanner, 20-second scan time with a $16-\mathrm{cm} \times 13-\mathrm{cm}$ field of view at a resolution of $0.3-\mathrm{mm}$ voxels, $120 \mathrm{kVp}, 3-8 \mathrm{~mA}$

11 (7 male and 4 Female) Age range: $16.45 \pm 5.76$ years

Graduate School, Faculty of Dentistry, Prince of Songkla University, for grant support teeth with maximum anchorage. Among them, one study was performed with self-ligated appliance, ${ }^{31}$ three studies used edgewise appliance $28,29,32$ and one study used $0.018 \times 0.025$-in Roth appliance. ${ }^{33}$ Four trials that did not use treatment with premolar extractions also showed reduction on bone thickness. Among them, one study compared self-ligated and conventional straight-wire appliances ${ }^{26}$ and did not find differences between the two techniques regarding buccal bone plate changes; one study compared two different types of self-ligated straight wire appliances - passive and active - and also did not find differences between groups ${ }^{27}$ and two studies used conventional Straight-wire appliances. ${ }^{30,34}$ Two studies didn't report the technique but described reduction in alveolar bone. ${ }^{35,36}$ One study reported extraction of maxillary first premolars and retraction of maxillary incisors, and showed no statistically significant differences of alveolar thickness before and after treatment. ${ }^{37}$ 
Table 2 - Methodological quality evaluation of included studies using Newcastle-Ottawa scale.

\begin{tabular}{|c|c|c|c|c|c|c|c|c|}
\hline & Study & $\begin{array}{l}\text { Ahn } \\
\text { et al. }\end{array}$ & $\begin{array}{l}\text { Nayak-Krishna } \\
\text { et al. }^{32}\end{array}$ & $\begin{array}{l}\text { Oliveira } \\
\text { et al. }{ }^{37}\end{array}$ & $\begin{array}{l}\text { Sarikaya } \\
\text { et al. }{ }^{33}\end{array}$ & $\begin{array}{l}\text { Thongudomporn } \\
\text { et al. }{ }^{34}\end{array}$ & $\begin{array}{l}\text { Uribe } \\
\text { et al. }{ }^{35}\end{array}$ & $\begin{array}{l}\text { Yodthong } \\
\text { et al. }{ }^{36}\end{array}$ \\
\hline \multirow{7}{*}{ Selection } & Sample size calculation & 0 & 0 & $\star$ & 0 & $\star$ & 0 & 0 \\
\hline & $\begin{array}{l}\text { Representativeness of } \\
\text { orthodontic patients }\end{array}$ & $\star$ & $\star$ & 0 & * & $\star$ & 0 & 0 \\
\hline & $\begin{array}{l}\text { Selection of the orthodontic } \\
\text { control group }\end{array}$ & 0 & 0 & 0 & 0 & 0 & 0 & 0 \\
\hline & $\begin{array}{l}\text { Ascertainment of the bone } \\
\text { before orthodontic treatment }\end{array}$ & * & * & $\star$ & $\star$ & $\star$ & $\star$ & * \\
\hline & $\begin{array}{l}\text { Outcome of interest not } \\
\text { present at the start }\end{array}$ & 0 & $\star$ & 0 & 0 & 0 & 0 & $\star$ \\
\hline & Training of assessors & 0 & 0 & 0 & 0 & 0 & 0 & 0 \\
\hline & $\begin{array}{l}\text { Description of inclusion/ } \\
\text { exclusion criteria }\end{array}$ & $\star$ & $\star$ & $\star$ & 0 & * & $\star$ & * \\
\hline \multirow{2}{*}{ Comparability } & $\begin{array}{c}\text { Comparability of groups on the } \\
\text { basis of the design }\end{array}$ & 0 & 0 & 0 & 0 & 0 & 0 & 0 \\
\hline & Management of confounders & $\star$ & $\star$ & $\star$ & 0 & 0 & $\star$ & $\star$ \\
\hline \multirow{3}{*}{ Outcome } & $\begin{array}{l}\text { Assessment of clinical } \\
\text { conditions }\end{array}$ & 0 & 0 & 0 & 0 & 0 & 0 & 0 \\
\hline & $\begin{array}{l}\text { Definitions and assessment } \\
\text { of bone resorption clearly } \\
\text { reported }\end{array}$ & $\star$ & $\star$ & $\star$ & 0 & $\star$ & $\star$ & $\star$ \\
\hline & $\begin{array}{l}\text { Adequacy of follow-up of } \\
\text { patients }\end{array}$ & 0 & 0 & 0 & 0 & 0 & 0 & 0 \\
\hline \multirow{2}{*}{ Statistics } & Validity of statistical analysis & $\star$ & $\star$ & $\star$ & $\star$ & $\star$ & $\star$ & $\star$ \\
\hline & Unit of analysis reported & $\star$ & 0 & 0 & 0 & 0 & $\star$ & 0 \\
\hline Total & $(14 / 14)$ & $7 / 14$ & $7 / 14$ & $6 / 14$ & $3 / 14$ & $6 / 14$ & $6 / 14$ & $6 / 14$ \\
\hline
\end{tabular}

\section{Different tooth evaluated}

Nine studies evaluated central and lateral incisors. ${ }^{29-37}$ Among them, eight studies evaluated maxillary incisors and three studies evaluated mandibular incisors. ${ }^{30,32,33}$ One study evaluated mandibular premolars, ${ }^{26}$ two studies evaluated maxillary premolars, ${ }^{27,28}$ two studies evaluated maxillary canines ${ }^{31,35}$ and one study evaluated mandibular molars. ${ }^{26}$

\section{Distance from CEJ}

Seven studies evaluated bone thickness changes at different distances from CEJ. Three studies evaluated bone changes at 3, 6 and $9 \mathrm{~mm}$ from CEJ. ${ }^{29,32,33}$ Two studies reported changes in alveolar thickness at crestal, midroot and apical areas, ${ }^{34,36}$ one study re- ported evaluation at 2, 4, 6, 8 and $10 \mathrm{~mm}^{\text {from } \mathrm{CEJ}^{35}}$ and one study reported evaluation at 2, 4, 6, 8, 10, 12 and $14 \mathrm{~mm}$ from CEJ. ${ }^{37}$

Regarding studies that evaluated the bone thickness on incisors at different levels from CEJ, only one study found a significant bone loss only at the cervical, ${ }^{33}$ while other studies found an increase in this region ${ }^{29,36}$. Some studies found bone loss at multiple sites. ${ }^{31,32,34,37}$ In addition, some studies reported bone loss at the buccal side of incisors, ${ }^{32,33}$ while others reported an increase in buccal bone..$^{29,36}$

Most of the studies showed that orthodontic treatment produces a reduction in bone thickness. ${ }^{26-28,31-35}$ The reduction in bone thickness was more pronounced at the palatal side, especially at incisors. 
Table 3 - Individual studies outcomes.

\begin{tabular}{|c|c|c|c|c|}
\hline Articles & Teeth & Groups & Views & Results* \\
\hline $\begin{array}{c}\text { Ahn et al. }{ }^{31}, \\
2013\end{array}$ & $\begin{array}{l}\text { Maxillary central } \\
\text { incisors } \\
\text { Maxillary lateral } \\
\text { incisors } \\
\text { Maxillary canines }\end{array}$ & $\begin{array}{l}\text { Test group (Class I } \\
\text { dentoalveolar protrusion } \\
\text { treated with extraction of } \\
\text { the four first premolars } \\
\text { and sliding mechanics } \\
\text { and straight-wire } \\
\text { appliance) }\end{array}$ & $\begin{array}{l}\text { Buccal side } \\
\text { Palatal side }\end{array}$ & 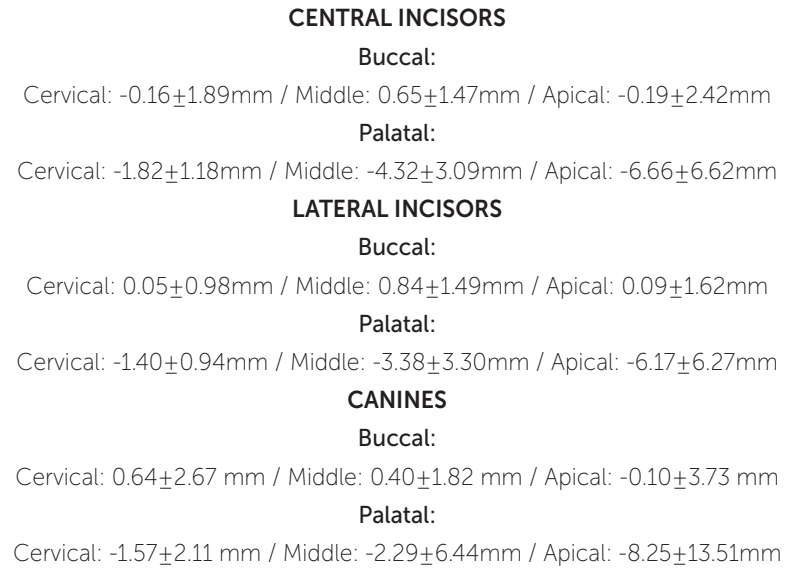 \\
\hline $\begin{array}{c}\text { Almeida et al. }{ }^{26} \text {, } \\
2015\end{array}$ & $\begin{array}{l}\text { Mandibular first } \\
\text { premolar } \\
\text { Mandibular } \\
\text { second premolar } \\
\text { Mandibular first } \\
\text { molar }\end{array}$ & $\begin{array}{l}\text { Test group (Class I } \\
\text { malocclusion treated } \\
\text { with self-ligated brackets) } \\
\text { Control group (Class I } \\
\text { malocclusion treated } \\
\text { with conventional } \\
\text { brackets) }\end{array}$ & $\begin{array}{l}\text { Buccal side at } \\
\text { apical height }\end{array}$ & $\begin{array}{l}\text { Test Group } \\
\text { First premolar: }-0.77 \pm 1.46 \mathrm{~mm} / \text { Second premolar: }-0.86 \pm 0.72 \mathrm{~mm} \\
\text { First molar: }-0.43 \pm 0.76 \mathrm{~mm} \\
\text { Control Group } \\
\text { First premolar: }-1.20 \pm 1.64 \mathrm{~mm} / \text { Second premolar: }-0.98 \pm 1.78 \mathrm{~mm} / \\
\text { First molar: }-0.55 \pm 0.91 \mathrm{~mm}\end{array}$ \\
\hline $\begin{array}{c}\text { Cattaneo et al. }{ }^{27}, \\
2011\end{array}$ & First premolar & $\begin{array}{l}\text { Test group (Damon } \\
\text { passive self-ligated } \\
\text { brackets) } \\
\text { Control group (In- } \\
\text { Ovation active self-ligated } \\
\text { brackets) }\end{array}$ & $\begin{array}{c}\text { Buccal cortical } \\
\text { bone plate }\end{array}$ & $\begin{array}{c}\text { Damon } T_{1}-T_{0}(n=32) \\
\text { Upper premolar: }-0.1 \pm 3.27 \mathrm{~mm} \\
\text { In-Ovation } T_{1}-T_{0}(n=32) \\
\text { Upper premolar: }-2.25 \pm 3.20 \mathrm{~mm}\end{array}$ \\
\hline $\begin{array}{l}\text { Lombardo et al. }{ }^{28} \text {, } \\
2013\end{array}$ & $\begin{array}{c}\text { Mandibular first } \\
\text { premolar } \\
\text { Maxillary second } \\
\text { premolar }\end{array}$ & $\begin{array}{l}\text { Test group (Class II } \\
\text { division } 1 \text { malocclusion } \\
\text { with extraction of the } \\
\text { upper first premolars and } \\
\text { lower second premolars } \\
\text { treated with Tweed } \\
\text { technique) } \\
\text { Control group } \\
\text { (orthodontic treatment } \\
\text { without extraction with } \\
\text { Tweed technique) }\end{array}$ & $\begin{array}{l}\text { Buccolingual } \\
\text { thickness (BT) }\end{array}$ & $\begin{array}{l}\text { Test group } \\
\text { First premolar BT changes: } 3.19 \mathrm{~mm} \\
\text { Second premolar BT changes: } 1.71 \mathrm{~mm} \\
\text { Control group } \\
\text { First premolar BT changes: } 0.98 \mathrm{~mm} \\
\text { Second premolar BT changes: } 0.67 \mathrm{~mm}\end{array}$ \\
\hline
\end{tabular}

\begin{tabular}{|c|c|c|c|c|}
\hline & & & & Maxillary central incisors BBT: \\
\hline & & & & $3 \mathrm{~mm}: 0.30 \pm 0.5 \mathrm{~mm} / 6 \mathrm{~mm}: 0.20 \pm 0.44 \mathrm{~mm} / 9 \mathrm{~mm}: 0.10 \pm 0.76 \mathrm{~mm}$ \\
\hline & & & & Maxillary central incisors LBT: \\
\hline & & & & $3 \mathrm{~mm}: 0.40 \pm 0.77 \mathrm{~mm} / 6 \mathrm{~mm}: 0.50 \pm 0.54 \mathrm{~mm} / 9 \mathrm{~mm}:-0.40 \pm 0.54 \mathrm{~mm}$ \\
\hline & Maxillary central & & $3 \mathrm{~mm}$ & Maxillary lateral incisors BBT: \\
\hline & incisors & Test group (patients with & $6 \mathrm{~mm}$ & $3 \mathrm{~mm}: 0.40 \pm 0.5 \mathrm{~mm} / 6 \mathrm{~mm}: 0.30 \pm 0.5 \mathrm{~mm} / 9 \mathrm{~mm}: 0.50 \pm 0.77 \mathrm{~mm}$ \\
\hline & Maxillary lateral & bimaxillary dentoalveolar & $9 \mathrm{~mm}$ & Maxillary lateral incisors LBT: \\
\hline Nayak-Krishna & incisors & protrusion treated & & $3 \mathrm{~mm}: 0.10 \pm 0.61 \mathrm{~mm} / 6 \mathrm{~mm}: 0.40 \pm 0.5 \mathrm{~mm} / 9 \mathrm{~mm}: 0.40 \pm 0.76 \mathrm{~mm}$ \\
\hline et al. ${ }^{32}, 2013$ & Mandibular & with extraction of first & Buccal bone & Mandibular lateral incisors BBT: \\
\hline & central incisors & premolars and edgewise & thickness (BBT) & $3 \mathrm{~mm}: 0.10 \pm 0.42 \mathrm{~mm} / 6 \mathrm{~mm}: 0.30 \pm 0.45 \mathrm{~mm} / 9 \mathrm{~mm}: 0.30 \pm 0.72 \mathrm{~mm}$ \\
\hline & Mandibular & technique) & Lingual bone & Mandibular lateral incisors LBT: \\
\hline & lateral incisors & & thickness (LBT) & $3 \mathrm{~mm}: 0.40 \pm 0.97 \mathrm{~mm} / 6 \mathrm{~mm}:-0.30 \pm 0.80 \mathrm{~mm} / 9 \mathrm{~mm}:-0.50 \pm 0.51 \mathrm{~mm}$ \\
\hline & & & & Mandibular central incisors BBT: \\
\hline & & & & $3 \mathrm{~mm}: 0.30 \pm 0.46 \mathrm{~mm} / 6 \mathrm{~mm}: 0.40 \pm 0.51 \mathrm{~mm} / 9 \mathrm{~mm}: 0.30 \pm 0.74 \mathrm{~mm}$ \\
\hline & & & & Mandibular central incisors LBT: \\
\hline & & & & $3 \mathrm{~mm}: 0.40 \pm 0.51 \mathrm{~mm} / 6 \mathrm{~mm}:-0.30 \pm 0.46 \mathrm{~mm} / 9 \mathrm{~mm}:-0.60 \pm 0.51 \mathrm{~mm}$ \\
\hline
\end{tabular}

* Difference between pretreatment and post treatment values as regards alveolar bone thickness. Negative values indicate a reduction in bone width; ML: midsagittal plane; RM $5 \mathrm{~mm}$ apart from ML to the right; RD $10 \mathrm{~mm}$ apart from ML to the right; LM 5mm apart from ML to the left; LD $10 \mathrm{~mm}$ apart from ML to the left. 
Table 3 - (Continuation) Individual studies outcomes.

\begin{tabular}{|c|c|c|c|c|}
\hline Articles & Teeth & Groups & Views & Results* \\
\hline $\begin{array}{l}\text { Oliveira et al. }{ }^{37}, \\
\qquad 2016\end{array}$ & $\begin{array}{c}\text { Maxillary central } \\
\text { and lateral } \\
\text { incisors }\end{array}$ & $\begin{array}{c}\text { Test group (Class II } \\
\text { division } 1 \text { and Class I } \\
\text { malocclusion treated } \\
\text { with extraction of first } \\
\text { premolars and edgewise } \\
\text { technique) }\end{array}$ & $\begin{array}{c}\text { Alveolar } \\
\text { bone width } \\
\text { measurements at } \\
2,4,6,8,10,12 \\
\text { and } 14 \mathrm{~mm} \text { apical } \\
\text { to the alveolar } \\
\text { crest }\end{array}$ & 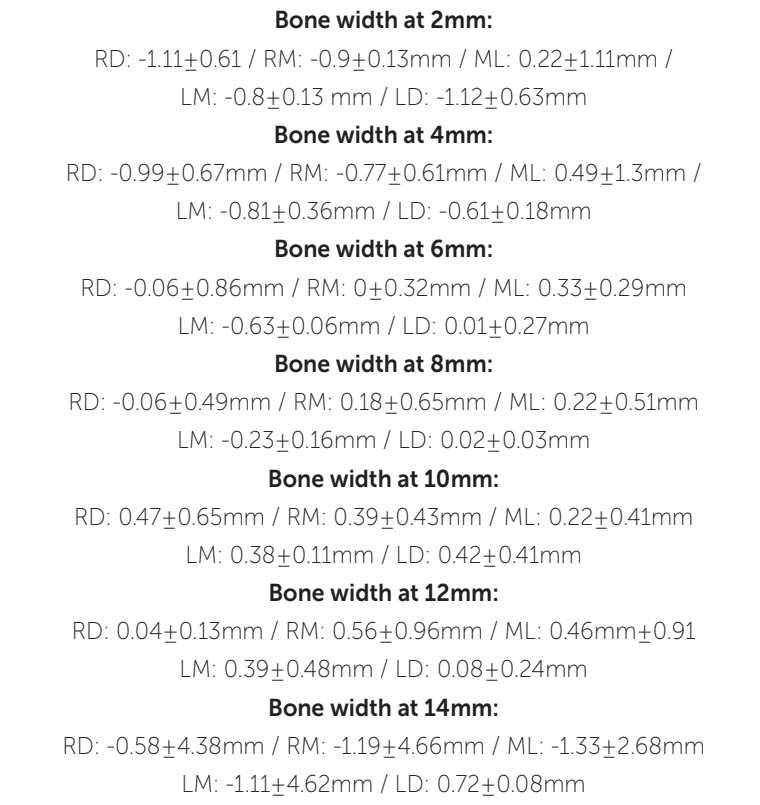 \\
\hline $\begin{array}{c}\text { Picanço et al. }{ }^{29} \text {, } \\
2013\end{array}$ & $\begin{array}{l}\text { Maxillary central } \\
\text { incisors }\end{array}$ & $\begin{array}{l}\text { Test group (Class II } \\
\text { malocclusion treated } \\
\text { with upper premolar } \\
\text { extraction) } \\
\text { Control group (Class I } \\
\text { and Class II malocclusion } \\
\text { treated without } \\
\text { extraction) }\end{array}$ & $\begin{array}{l}\text { Buccal and palatal } \\
\text { sides at } 3 \mathrm{~mm} \text {, } \\
6 \mathrm{~mm} \text { and } 9 \mathrm{~mm} \\
\text { from cemento- } \\
\text { enamel junction } \\
\text { UL: Buccal } \\
\text { alveolar bone } \\
\text { UP: Palatal alveolar } \\
\text { bone }\end{array}$ & 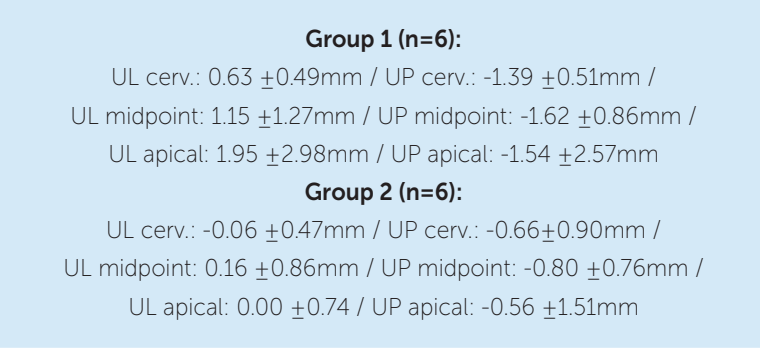 \\
\hline $\begin{array}{l}\text { Sarikaya et al. }{ }^{33}, \\
2002\end{array}$ & $\begin{array}{l}\text { Maxillary central } \\
\text { incisors } \\
\text { Maxillary lateral } \\
\text { incisors } \\
\text { Mandibular } \\
\text { central incisors } \\
\text { Mandibular } \\
\text { lateral incisors }\end{array}$ & $\begin{array}{l}\text { Test group (patients with } \\
\text { dentoalveolar bimaxillary } \\
\text { protrusion treated with } \\
\text { extractions of the } 4 \text { first } \\
\text { premolars and straight- } \\
\text { wire appliances) }\end{array}$ & $\begin{array}{l}\text { Buccal and palatal } \\
\text { sides at } 3 \mathrm{~mm} \text {, } \\
6 \mathrm{~mm} \text { and } 9 \mathrm{~mm} \\
\text { from cemento- } \\
\text { enamel junction }\end{array}$ & 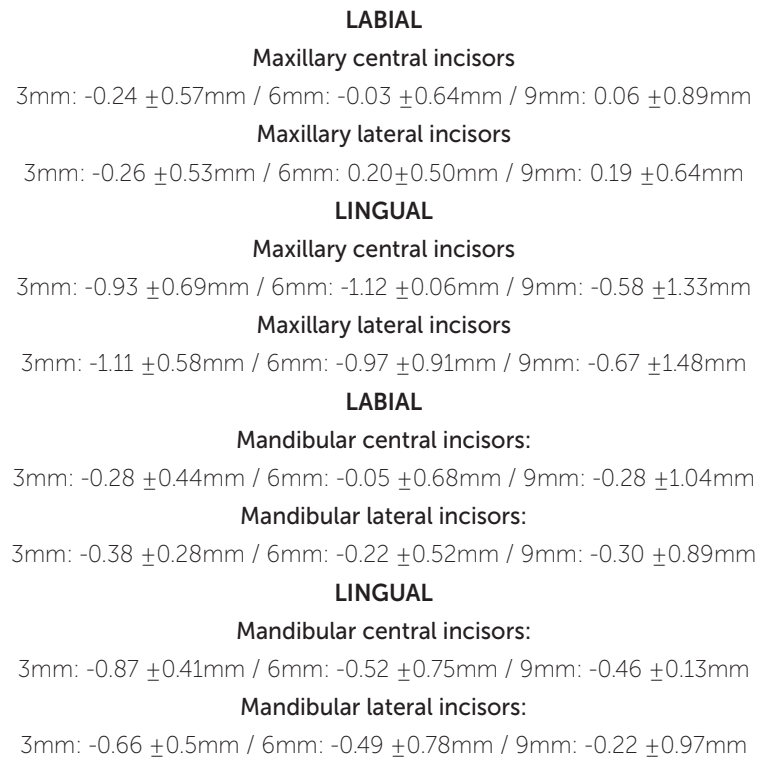 \\
\hline $\begin{array}{c}\text { Sun et al. }{ }^{30} \text {, } \\
2015\end{array}$ & $\begin{array}{l}\text { Mandibular } \\
\text { incisors }\end{array}$ & $\begin{array}{l}\text { Test group (patients with } \\
\text { Class III malocclusion } \\
\text { treated with straight-wire } \\
\text { appliance) } \\
\text { Control group (patients } \\
\text { with normal occlusion) }\end{array}$ & $\begin{array}{l}\text { Labial alveolar } \\
\text { bone thickness at } \\
\text { apical level } \\
\text { Lingual alveolar } \\
\text { bone thickness at } \\
\text { apical level }\end{array}$ & $\begin{array}{l}\text { Labial alveolar bone thickness: } 1.71 \pm 0.43 \mathrm{~mm} \\
\text { Lingual alveolar bone thickness: }-2.07 \pm 0.51 \mathrm{~mm}\end{array}$ \\
\hline
\end{tabular}

* Difference between pretreatment and post treatment values as regards alveolar bone thickness. Negative values indicate a reduction in bone width; ML: midsagittal plane; RM $5 \mathrm{~mm}$ apart from ML to the right; RD $10 \mathrm{~mm}$ apart from ML to the right; LM 5mm apart from ML to the left; LD $10 \mathrm{~mm}$ apart from ML to the left. 
Table 3 - (Continuation) Individual studies outcomes.

\begin{tabular}{|c|c|c|c|c|}
\hline Articles & Teeth & Groups & Views & Results* \\
\hline $\begin{array}{c}\text { Thongudomporn } \\
\text { et al. }{ }^{34}, 2015\end{array}$ & Maxillary incisors & $\begin{array}{l}\text { Test group (patients with } \\
\text { mild skeletal Class III and } \\
\text { straight-wire appliances) }\end{array}$ & $\begin{array}{l}\text { Buccal and palatal } \\
\text { sides at } 3 \mathrm{~mm} \text {, } \\
6 \mathrm{~mm} \text { and } 9 \mathrm{~mm} \\
\text { from cemento- } \\
\text { enamel junction }\end{array}$ & $\begin{array}{l}\text { Labial alveolar thickness: } \\
3 \mathrm{~mm}:-0.12 \pm 0.18 \mathrm{~mm} / 6 \mathrm{~mm}:-0.18 \pm 0.31 \mathrm{~mm} / 9 \mathrm{~mm}:-0.01 \pm 0.63 \mathrm{~mm} \\
\text { Palatal alveolar thickness: } \\
3 \mathrm{~mm}:-0.13 \pm 0.24 \mathrm{~mm} / 6 \mathrm{~mm}:-0.34 \pm 0.30 \mathrm{~mm} / 9 \mathrm{~mm}:-0.59 \pm 0.48 \mathrm{~mm}\end{array}$ \\
\hline $\begin{array}{l}\text { Uribe et al. }{ }^{35} \text {, } \\
\qquad 2013\end{array}$ & $\begin{array}{l}\text { Maxillary central } \\
\text { incisors } \\
\text { Maxillary canines }\end{array}$ & $\begin{array}{l}\text { Test group (patients with } \\
\text { unilaterally or bilaterally } \\
\text { congenitally missing } \\
\text { maxillary lateral incisors) }\end{array}$ & $\begin{array}{l}\text { Alveolar } \\
\text { bone width } \\
\text { measurements } \\
\text { at } 2,4,6,8 \text {, and } \\
10 \mathrm{~mm} \text { apical to } \\
\text { the alveolar crest }\end{array}$ & $\begin{array}{c}\text { Central incisor: } \\
2 \mathrm{~mm}:-0.45 \pm 0.55 \mathrm{~mm} / 4 \mathrm{~mm}:-0.55 \pm 0.76 \mathrm{~mm} / 6 \mathrm{~mm}:-0.88 \pm 1.13 \mathrm{~mm} \\
8 \mathrm{~mm}:-1.35 \pm 1.22 \mathrm{~mm} / 10 \mathrm{~mm}:-1.29 \pm 1.64 \mathrm{~mm} \\
\text { Canine: } \\
2 \mathrm{mm:}-0.80 \pm 1.17 \mathrm{~mm} / 4 \mathrm{~mm}:-0.67 \pm 1.12 \mathrm{~mm} / 6 \mathrm{~mm}:-0.29 \pm 1.27 \mathrm{~mm} \\
8 \mathrm{~mm}:-0.01 \pm 1.43 \mathrm{~mm} / 10 \mathrm{~mm}:-0.07 \pm 1.59 \mathrm{~mm}\end{array}$ \\
\hline $\begin{array}{c}\text { Yodthong et al. }{ }^{36} \\
2013\end{array}$ & Maxillary incisors & $\begin{array}{l}\text { Test group (patients } \\
\text { receiving orthodontic } \\
\text { treatment with upper } \\
\text { incisors bound to } \\
\text { retraction with a space } \\
>4 \text { mm between lateral } \\
\text { incisors and canines }\end{array}$ & $\begin{array}{l}\text { Labial alveolar } \\
\text { thickness and } \\
\text { palatal alveolar } \\
\text { thickness at } \\
\text { crestal, midroot } \\
\text { and apical levels. }\end{array}$ & $\begin{array}{l}\text { Labial thickness at crestal level: }-0.4 \pm 0.3 \mathrm{~mm} \\
\text { Palatal thickness at crestal level: } 0.20 \pm 0.36 \mathrm{~mm} \\
\text { Labial thickness at midroot level: }-0.2 \pm 0.3 \mathrm{~mm} \\
\text { Palatal thickness at midroot level: }-0.1 \pm 0.60 \mathrm{~mm} \\
\text { Labial thickness at apical level: } 0 \pm 0.3 \mathrm{~mm} \\
\text { Palatal thickness at apical level: }-0.6 \pm 1.41 \mathrm{~mm}\end{array}$ \\
\hline
\end{tabular}

* Difference between pretreatment and post treatment values as regards alveolar bone thickness. Negative values indicate a reduction in bone width; ML: midsagittal plane; RM 5mm apart from ML to the right; RD $10 \mathrm{~mm}$ apart from ML to the right; LM 5mm apart from ML to the left; LD $10 \mathrm{~mm}$ apart from ML to the left

\section{DISCUSSION}

In spite of many studies investigating the association of bone resorptions and orthodontic treatment, this is the first systematic review to assess the effects of orthodontic treatment on bone remodeling. Although the 12 selected studies are very heterogeneous, it can be observed that most of the studies showed that orthodontic treatment produces a reduction in bone thickness. No meta-analysis could be performed because the studies included different orthodontic techniques, evaluated distinct teeth with diverse forces. Furthermore, variable treatment times were found.

Studies that performed measurements of bone remodeling without the usage of CBCT were excluded. CBCT enables examination of alveolar bone morphology with quality, since three-dimensional images are not subject to distortion or superimposition..$^{23,24}$

Regarding the specification of the force used in tooth movement, the only authors who reported the measurement of force used in orthodontic treatment were: Nayak-Krishna et al, ${ }^{32}$ who reported light continuous forces of $100 \mathrm{~g}$ for retraction of anterior teeth; Ahn et $\mathrm{al},{ }^{31}$ who reported $200 \mathrm{~g}$ of force on elastic chains also to retract anterior teeth; Thongudomporn et al, ${ }^{34}$ who placed $89.6 \mathrm{~g}$ on upper incisors for buccal tipping movements; and Oliveira et al, ${ }^{37}$ who activated the incisors retraction with a force of $150 \mathrm{~g}$ per side. The individual analysis of the selected studies does not imply that a specific type of force causes more alveolar bone loss than others.

The articles were heterogeneous regarding the type of orthodontic movement performed. As to the treatment plan, some articles reported only alignment and leveling movements ${ }^{26,27,30}$ and other studies performed premolars extractions with retraction of the anterior teeth. ${ }^{28-33,36,37}$ Thongudomporn et al ${ }^{34}$ treated patients with anterior crossbite through buccal tipping and extrusion of upper incisors using advancing loops and Class III elastics.

The heterogeneity of the studies also comprised the type of orthodontic bracket and technique; thus few studies used Straight-wire technique, ${ }^{26,27,30,31,33,34}$ while others performed Edgewise technique $28,29,32,37$. Almeida et al. ${ }^{26}$ compared self-ligating with conventional brackets, while Cattaneo et al. ${ }^{27}$ compared different types of self-ligating brackets. The heterogeneity and the quality of the included studies are the limitations of this study.

Regarding the regions where bone changes were measured, the following sites were analyzed: alveolar bone area at cervical, middle and apical levels, ${ }^{29,31-34}$ most external proeminence of the buccal bone in 
the most apical portion of the root, ${ }^{26}$ alveolar bone width measurements at 2, 4, 6, 8, 10, 12 and $14 \mathrm{~mm}$ apical to the alveolar crest ${ }^{35,37}$ and labial and palatal bone thickness at the crestal level, midroot level and apical level. ${ }^{36}$

The absence or insufficiency of alveolar bone thickness is a complicating factor for orthodontic treatment. The occurrence of dehiscences and fenestrations during orthodontic treatment depends on factors such as: direction of movement, frequency and magnitude of orthodontic forces, volume and anatomical integrity of periodontal tissues. ${ }^{4}$

As regards the implications for dental practice, we consider that these findings are relevant and have to be considered not only in diagnosis but also in the planning of tooth movement, in order to prevent the occurrence of dehiscence and fenestration in the alveolar bone. Additionally, it is interesting to notice that the majority of the studies observed a higher percent- age of bone remodeling at the palatal side. Probably, the reason for this greater effect at the palatal side is a result of the type of orthodontic movement (retraction of the incisors). ${ }^{33}$ The loss of alveolar bone at the palatal side doesn't have an impact on esthetics, but it has to be considered on the orthodontic treatment, since if the patient does not undergo a rigid periodontal follow-up it can result in severe and definitive loss of periodontal support. We emphasize the need for periodontal diagnosis; strict dental biofilm control and regular maintenance visits for patients undergoing orthodontic treatment.

\section{CONCLUSION}

On patients undergoing different orthodontic treatment techniques, there was a significant bone thickness reduction, mainly on the palatal side. However, the results should be interpreted with caution, because of the heterogeneity of the included studies.

\section{Authors contribution (ORCID ${ }^{(1)}$ )}

Michelle Sendyk (MS): 0000-0001-9850-8790

Daniele Sigal Linhares (DSL): 0000-0003-1979-1343

Claudio M. Pannuti (CMP): 0000-0003-4181-3975 (10)

João Batista de Paiva (JBP): 0000-0002-3178-4843

José Rino Neto (JRN): 0000-0002-9124-8698

Conception or design of the study: MS, CMP. Data acquisition, analysis or interpretation: MS, DSL, CMP, CMP, JBP, JRN. Writing the article: MS. Critical revision of the article: MS, DSL, CMP, CMP, JBP, JRN. Final approval of the article: MS, DSL, CMP, CMP, JBP, JRN. Overall responsibility: MS. 


\section{REFERENCES}

1. Ericsson I, Thilander B, Lindhe J, Okamoto $\mathrm{H}$. The effect of orthodontic tilting movements on the periodontal tissues of infected and non-infected dentitions in dogs. J Clin Periodontol. 1977 Nov:4(4):278-93.

2. Gorbunkova A, Pagni G, Brizhak A, Farronato G, Rasperini G. Impact of orthodontic treatment on periodontal tissues: a narrative review of multidisciplinary literature. Int J Dent. 2016;2016:4723589.

3. Bollen AM, Cunha-Cruz J, Bakko DW, Huang GJ, Hujoel PP. The effects of orthodontic therapy on periodontal health: a systematic review of controlled evidence. J Am Dent Assoc. 2008 Apr;139(4):413-22.

4. Enhos S, Uysal T, Yagci A, Veli I, Ucar Fl, Ozer T. Dehiscence and fenestration in patients with different vertical growth patterns assessed with cone-beam computed tomography. Angle Orthod. 2012 Sept;82(5):868-74

5. Handelman CS. The anterior alveolus: its importance in limiting orthodontic treatment and its influence on the occurrence of iatrogenic sequelae. Angle Orthod. 1996:66(2):95-109; discussion 109-10.

6. Proffit WR, Ackerman JL. Diagnosis and treatment planning. In: Current orthodontic concepts and techniques. St. Louis: Mosby; 1982.

7. Mulie RM, Hoeve AT. The limitations of tooth movement within the symphysis, studied with laminagraphy and standardized occlusal films. J Clin Orthod. 1976 Dec;10(12):882-93, 886-9.

8. Baumgaertel S, Hans MG. Buccal cortical bone thickness for mini-implant placement. Am J Orthod Dentofacial Orthop. 2009 Aug:136(2):230-5

9. Poggio PM, Incorvati C, Velo S, Carano A. "Safe zones": a guide for miniscrew positioning in the maxillary and mandibular arch. Angle Orthod 2006 Mar;76(2):191-7.

10. Agrawal N, Kundu D, Agrawal K, Singhal A. Comparison of longitudinal changes in clinical periodontal parameters of canines and first molars treated with fixed orthodontic appliances. Am J Orthod Dentofacial Orthop. 2016 Mar;149(3):325-30

11. Garlock DT, Buschang PH, Araujo EA, Behrents RG, Kim KB. Evaluation of marginal alveolar bone in the anterior mandible with pretreatment and posttreatment computed tomography in nonextraction patients. Am J Orthod Dentofacial Orthop. 2016 Feb;149(2):192-201.

12. Castro LO, Castro IO, Alencar AH, Valladares-Neto J, Estrela C. Cone beam computed tomography evaluation of distance from cementoenamel junction to alveolar crest before and after nonextraction orthodontic treatment. Angle Orthod. 2016 July: 86(4):543-9.

13. Hu KS, Kang MK, Kim TW, Kim KH, Kim HJ. Relationships between dental roots and surrounding tissues for orthodontic miniscrew installation. Angle Orthod. 2009 Jan;79(1):37-45

14. Engelking G, Zachrisson BU. Effects of incisor repositioning on monkey periodontium after expansion through the cortical plate. Am J Orthod. 1982 July:82(1):23-32.

15. Thilander B, Nyman S, Karring T, Magnusson I. Bone regeneration in alveolar bone dehiscences related to orthodontic tooth movements. Eur J Orthod. 1983 May:5(2):105-14

16. Ten Hoeve A, Mulie RM. The effect of antero-postero incisor repositioning on the palatal cortex as studied with laminagraphy. J Clin Orthod. 1976 Nov:10(11):804-22

17. Edwards JG. A study of the anterior portion of the palate as it relates to orthodontic therapy. Am J Orthod. 1976 Mar;69(3):249-73.

18. Tong H, Enciso R, Van Elslande D, Major PW, Sameshima GT. A new method to measure mesiodistal angulation and faciolingual inclination of each whole tooth with volumetric cone-beam computed tomography images. Am J Orthod Dentofacial Orthop. 2012 July:142(1):133-43

19. Podesser B, Williams S, Bantleon HP, Imhof H. Quantitation of transverse maxillary dimensions using computed tomography: a methodological and reproducibility study. Eur J Orthod. 2004 Apr;26(2):209-15.

20. Masumoto T, Hayashi I, Kawamura A, Tanaka K, Kasai K. Relationships among facial type, buccolingual molar inclination, and cortical bone thickness of the mandible. Eur J Orthod. 2001 Feb:23(1):15-23.
21. Mitra S, Ravi MS. Evaluation of buccolingual inclination of posterior teeth in different facial patterns using computed tomography. Indian J Dent Res 2011 May-June:22(3):376-80

22. Shewinvanakitkul W, Hans MG, Narendran S, Martin Palomo J. Measuring buccolingual inclination of mandibular canines and first molars using CBCT. Orthod Craniofac Res. 2011 Aug:14(3):168-74.

23. Lund H, Grondahl K, Grondahl HG. Cone beam computed tomography for assessment of root length and marginal bone level during orthodontic treatment. Angle Orthod. 2010 May:80(3):466-73.

24. Timock AM, Cook V, McDonald T, Leo MC, Crowe J, Benninger BL, et al. Accuracy and reliability of buccal bone height and thickness measurements from cone-beam computed tomography imaging. Am J Orthod Dentofacial Orthop. 2011 Nov;140(5):734-44.

25. Chambrone L, Chambrone LA, Lima LA. Effects of occlusal overload on peri-implant tissue health: a systematic review of animal-model studies. J Periodontol. 2010 Oct;81(10):1367-78.

26. Almeida MR, Futagami C, Conti AC, Oltramari-Navarro PV, Navarro RL. Dentoalveolar mandibular changes with self-ligating versus conventional bracket systems: A CBCT and dental cast study. Dental Press J Orthod. 2015 May-June:20(3):50-7

27. Cattaneo PM, Treccani M, Carlsson K, Thorgeirsson T, Myrda A, Cevidanes LH, et al. Transversal maxillary dento-alveolar changes in patients treated with active and passive self-ligating brackets: a randomized clinical trial using CBCT-scans and digital models. Orthod Craniofac Res. 2011 Nov:14(4):222-33.

28. Lombardo L, Bragazzi R, Perissinotto C, Mirabella D, Siciliani G. Cone-beam computed tomography evaluation of periodontal and bone support loss in extraction cases. Prog Orthod. 2013 Sept 11:14:29.

29. Picanço PR, Valarelli FP, Cançado RH, Freitas KM, Picanço GV. Comparison of the changes of alveolar bone thickness in maxillary incisor area in extraction and non-extraction cases: computerized tomography evaluation. Dental Press J Orthod. 2013 Sept-Oct;18(5):91-8.

30. Sun B, Tang J, Xiao P, Ding Y. Presurgical orthodontic decompensation alters alveolar bone condition around mandibular incisors in adults with skeletal Class III malocclusion. Int J Clin Exp Med. 2015;8(8):12866-73.

31. Ahn HW, Moon SC, Baek SH. Morphometric evaluation of changes in the alveolar bone and roots of the maxillary anterior teeth before and after en masse retraction using cone-beam computed tomography. Angle Orthod. 2013 Mar:83(2):212-21.

32. Nayak Krishna US, Shetty A, Girija MP, Nayak R. Changes in alveolar bone thickness due to retraction of anterior teeth during orthodontic treatment: a cephalometric and computed tomography comparative study. Indian J Dent Res. 2013:24(6):736-41.

33. Sarikaya S, Haydar B, Ciğer S, Ariyürek M. Changes in alveolar bone thickness due to retraction of anterior teeth. Am J Orthod Dentofacial Orthop. 2002 July:122(1):15-26.

34. Thongudomporn U, Charoemratrote C, Jearapongpakorn S. Changes of anterior maxillary alveolar bone thickness following incisor proclination and extrusion. Angle Orthod. 2015 July: 85(4):549-54

35. Uribe F, Padala S, Allareddy V, Nanda R. Cone-beam computed tomography evaluation of alveolar ridge width and height changes after orthodontic space opening in patients with congenitally missing maxillary lateral incisors. Am J Orthod Dentofacial Orthop. 2013 Dec;144(6):848-59.

36. Yodthong N, Charoemratrote C, Leethanakul C. Factors related to alveolar bone thickness during upper incisor retraction. Angle Orthod. 2013 May:83(3):394-401.

37. Oliveira TMF, Claudino LV, Mattos CT, Sant'Anna EF. Maxillary dentoalveolar assessment following retraction of maxillary incisors: a preliminary study Dental Press J Orthod. 2016 Sept-Oct:21(5):82-9. 